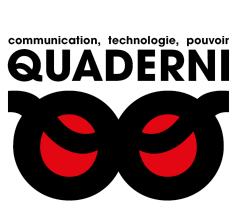

\title{
Quaderni
}

Communication, technologies, pouvoir

98 | Hiver 2018-2019

Humanités numériques : vers l'institutionnalisation

\section{Le projet E-SIGNA, le numérique au service des sources et des documents de l'emblématique médiévale}

\section{Matteo Ferrari et Laurent Hablot}

\section{(2) OpenEdition \\ Journals}

Édition électronique

URL : https://journals.openedition.org/quaderni/1469

DOI : 10.4000 /quaderni.1469

ISSN : 2105-2956

Éditeur

Les éditions de la Maison des sciences de l'Homme

Édition imprimée

Date de publication : 5 février 2019

Pagination : 107-122

Référence électronique

Matteo Ferrari et Laurent Hablot, «Le projet E-SIGNA, le numérique au service des sources et des documents de l'emblématique médiévale », Quaderni [En ligne], 98 | Hiver 2018-2019, mis en ligne le 05 février 2021, consulté le 08 janvier 2022. URL : http://journals.openedition.org/quaderni/1469 ; DOI : https://doi.org/10.4000/quaderni.1469 


\section{$D$ ossier}

\section{Le projet E-SIGNA,}

le numérique

au service des

sources et des

documents de

\section{l'emblématique médiévale}

\section{Matteo \\ Ferrari}

Paris, EPHE - Poitiers, CESCM - Namur, PraME

\section{Laurent Hablot}

Paris EPHE -PSL - SAPRAT
À partir du milieu du XII ${ }^{\mathrm{e}}$ siècle, dans toute la société européenne l'expression de l'identité passe aussi par l'usage de signes figurés capables de représenter une personne, physique ou morale, ou un groupe de personnes. Armoiries et signes para-héraldiques (cimiers, tenants, enseignes de fonction), devises, mots emblématiques se multiplient et envahissent l'espace, reproduits sur toute sorte de supports. Parfois également chargés d'une portée symbolique, en fonction de leur disposition dans l'espace, des circonstances d'emploi et de leur association à d'autres signes analogues ou à des images d'autre type, les représentations héraldiques et emblématiques offrent à leurs utilisateurs la possibilité d'exprimer leur rang, leurs fonctions, leurs droits et prétentions, leurs liens sociaux, politiques et lignagers ; de marquer une propriété ; d'afficher une culture, des valeurs, des idéaux ${ }^{1}$.

Depuis la fin des années 1970, les spécialistes d'emblématique, et notamment d'héraldique, ont cherché à intégrer l'emploi d'outils informatiques dans leur activité de recherche, saisissant les avantages, en termes de connaissances nouvelles, que l'application de ces instruments nouveaux pouvait porter. Leurs espoirs reposaient essentiellement dans l'élaboration d'un système d'encodage pour la description des armoiries (blasonnement) et dans la création d'un logiciel capable de stocker, classer et rendre exploitables les informations relatives aux centaines de milliers d'emblèmes connus dans toute l'Europe. Le but était de mettre à disposition un répertoire exhaustif qui aurait permis, à terme, d'identifier toute sorte d'armoiries de manière automatisée, rapide et sûre ${ }^{2}$. Les résultats atteints par ces pionniers de l'emblématique numérique n'ont pas été 
toutefois à la hauteur des attentes. Développées par des chercheurs ou des passionnés avertis travaillant de manière solitaire, ces premières bases de données rassemblaient en effet des corpus partiels et résultats difficilement exploitables par l'ensemble de la communauté scientifique ${ }^{3}$.

Dans les dernières années le débat autour de l'application du numérique à l'emblématique, notamment à celle médiévale, a pourtant repris force. Les chercheurs dans ce domaine participent ainsi pleinement au développement des humanités numériques avec des résultats encourageants. Si la création de logiciels capables d'identifier les armoiries de manière automatisée reste à l'ordre du jour, même si leur construction repose désormais sur de nouvelles bases ${ }^{4}$, de nos jours plusieurs centres européens de recherche et de conservation développent également des projets associant le numérique à l'étude des formes et des fonctions de l'emblématique médiévale. Ces initiatives visent principalement, d'un côté, à répertorier, décrire et analyser des corpus emblématiques spécifiques (collections de sceaux et de manuscrits, ensembles d'armoiries, catalogues d'érudits) ${ }^{5}$ et à les rendre interrogeables par le biais de logiciels ; de l'autre, à numériser et à rendre plus facilement identifiables et exploitables les sources et les outils bibliographiques concernant l'héraldique médiévale et moderne (armoriaux, traités héraldiques, études d'érudits) ${ }^{6}$.

Le projet E-SIGNA, porté par Laurent Hablot (Paris, EPHE-Saprat), s'inscrit dans cette tradition d'études qui couple l'emblématique aux humanités numériques, tout en la rénovant par son approche à la matière et par ses objectifs.
E-SIGNA réunit trois programmes de recherche préexistants qui ont été initialement développés, sous des modalités différentes, au CESCM de Poitiers à partir de l'année 2012. Centrés sur le thème de l'emblématique médiévale et moderne et combinés à trois bases de données et de connaissances en ligne, déjà accessibles au public depuis quelques années, ils associent la rigueur de l'érudition classique aux possibilités heuristiques et de communication offertes par les nouvelles technologies : Devise, recensant les emblèmes utilisés par les élites européennes entre la fin du Moyen Âge et le début de l'époque moderne ; Sigilla, visant à répertorier les sceaux conservés dans les collections françaises; ArmmA, dédiée à l'inventaire et à l'étude des décors héraldiques monumentaux produits dans la France médiévale.

E-SIGNA a été donc créé à l'automne 2017 avec le but de pérenniser, de mutualiser et d'actualiser ces programmes de recherche et les bases qui y sont associées. Il se propose d'assurer le développement de ces dernières et leur interopérabilité, interne et externe, et d'en promouvoir la visibilité dans le cadre du Domaine d'intérêt majeur (DIM) de l'Île-de-France « Sciences du texte et connaissances nouvelles ", auquel il a été intégré au printemps 2018. Autonomes mais complémentaires, les projets ArmmA, Devise et Sigilla se signalent par leur approche originelle à l'étude de l'emblématique médiévale, par leur extension géographique et chronologique, par la méthodologie d'analyse employée, par l'élaboration d'outils informatiques novateurs et accessibles également par des non-spécialistes, par leurs objectifs qui couplent la recherche scientifique à la mise en valeur du patrimoine artistique, historique, documentaire. 
DEVISE : emblématique et héraldique à la fin du Moyen Âge

Mise en ligne en 2012, la base Devise constitue l'extension naturelle des recherches de thèse de doctorat que Laurent Hablot a consacrées au thème de la devise, un système emblématique qui se développe dans les cours princières à partir des années 1340 et qui marque les élites européennes jusqu'à la pleine Renaissance ${ }^{7}$. Entretenant des liens étroits avec le système héraldique, les devises se posent comme complémentaires à l'armoirie, dont elles se différencient toutefois par leur forme-l'abstraction du signe héraldique laisse en effet ici la place à des représentations bien plus réalistes - et par leur fonction. Librement choisies par la personne qui les porte et, donc, strictement attachées à elle, les devises sont aussi un signe politique et militaire que les membres des élites peuvent partager avec leurs fidèles ${ }^{8}$.

Développée en partenariat avec le service informatique I-Media de l'Université de Poitiers avec un logiciel Lodel et actuellement stockée sur les serveurs de cette Université, la base DEVISE a donc pour ambition de réunir l'ensemble des données relatives aux emblèmes utilisés par les élites européennes durant la fin du Moyen Âge et la première Renaissance (1350-1550). Elle s'appuie sur le déjà vaste corpus rassemblé par Laurent Hablot dans sa thèse (plus d'un millier de notices), enrichi par les nombreuses informations ajoutées depuis plus de quinze ans grâce à de nouvelles recherches et à de très fructueuses collaborations à travers le monde. DEVISE a été d'ailleurs conçue dans la forme d'un projet collaboratif, permettant aux chercheurs qui le souhaitent de s'associer au travail d'inventaire, à la fois signalant des occurrences non encore répertoriées, corrigeant ou complétant les notices déjà en ligne ou, encore, en en rédigeant des nouvelles ${ }^{10}$. Dans ce but, les utilisateurs de DEVISE peuvent contacter à tout moment l'auteur de la base, à l'adresse internet indiqué dans la page contenant le descriptif du projet. L'alimentation collaborative de la base a été déjà testée, avec succès, grâce aux financements obtenus de l'Université d'Heidelberg et du consortium Cosme qui ont permis d'associer au travail, même si sur une courte durée, de jeunes chercheurs français et étrangers.

Les collaborations établies ont ainsi facilité le développement de la base, qui compte à ce jour un millier de notices environ. Celles-ci sont consacrées à l'emblématique des membres d'une soixantaine de familles parmi les plus importantes de la France et de l'Europe entre la fin du Moyen Âge et le début de l'époque moderne (AnjouNaples, Aragon, Bavière, Bourbon, Bourgogne, Bretagne, Castille, Lancastre, Luxembourg SaintPol, Savoie, Valois, Visconti...).

Chaque emblème adopté par un personnage fait l'objet d'une notice spécifique. Introduite par un intitulé portant l'appellation courante de la devise (la cordelière de Pierre II de Bretagne, le fusil de Philippe le Bon, la colombina ou le tizzone des Visconti) et sa description sommaire, et précédée par des entrées générant des index (chronologie, aire d'emploi, nom de l'utilisateur et famille d'appartenance, type de devise, mot emblématique éventuellement associé), la notice propose une interprétation de la devise en rapport avec le caractère et la culture de son possesseur 
et des circonstances historiques d'adoption. Ce travail herméneutique s'appuie sur un corpus - si possible exhaustif - d'attestations visuelles et écrites, indiquées avec précision (des liens hypertexte sont utilisés pour renvoyer le lecteur vers les documents publiés en ligne). La notice est par ailleurs accompagnée par des images sélectionnées, aptes également à illustrer des applications concrètes de la devise examinée. En revanche, des reconstructions graphiques sont souvent proposées quand celle-ci ne peut pas (ou ne peut plus) être documentée par des attestations matérielles (c'est le cas, par exemple, des devises de Valentina Visconti, connues essentiellement d'après les sources écrites). Une bibliographie et un apparat de notes essentiels complètent l'étude. Enfin, chaque notice est liée à une fiche personnage par le biais d'une notice d'autorité qui donne les informations généalogiques (date de mort, fonctions recouvertes, noms des parents, de l'épouse et des enfants) et emblématiques principales (blasonnement de l'armoirie, appartenance à un ordre de chevalerie) relatives au porteur de la devise. Cette solution permet ainsi de reconstituer assez aisément la panoplie emblématique d'un personnage et d'en suivre l'évolution dans le temps.

Les recherches à l'intérieur de la base peuvent être effectuées de manière assez intuitive, via le menu affiché sur la page d'accueil. Elles sont possibles soit par emblème, soit par famille. La première option permet de réaliser des recherches à l'intérieur d'index organisés sous forme de listes (ordonnées suivant l'ordre alphabétique ou chronologique) consacrées aux personnages, aux familles, aux aires géographiques et aux périodes d'emploi (ceux-ci établis sur la base d'intervalles de dix ans), mais également aux devises, mots, lettres et couleurs emblématiques. Cette dernière option permet à la fois d'identifier plus facilement les emblèmes qu'un utilisateur pourrait rechercher et de les associer directement à leur propriétaire, mais également d'avoir un aperçu sur des devises partagées au sein des élites européennes (c'est le cas, par exemple, de la ceinture avec le mot « esperance » que Louis II de Bourbon partage avec ses proches et ses parents). La recherche par famille, en revanche, donne accès d'abord à la liste des personnages qui, pour chaque lignage, sont connus pour avoir fait usage d'une ou de plusieurs devises et, ensuite, au répertoire des emblèmes qu'il a utilisés ${ }^{11}$.

Sans perdre sa dimension originale, européenne et collaborative, dans le cadre du plan lancé par le projet E-SIGNA à l'intérieur du DIM « Science $\mathrm{du}$ texte et connaissances nouvelles », le projet Devise portera en priorité sur les collections de manuscrits des bibliothèques de l'île-de-France et sur les grandes collections médiévales de la région. Des contacts avec les institutions préposées à la conservation du patrimoine manuscrit (Bibliothèque nationale de France, Bibliothèque de l'Arsenal, Musées du Louvre et de Cluny) existent déjà ou seront mis rapidement en place. Il sera également question de renforcer les liens et les échanges déjà opérationnels avec d'autres bases concernées par ces données, telle Bibale consacrée à la reconstruction des collections anciennes de manuscrits et à leur transmission ( $<$ http://bibale.irht.cnrs.fr/ $>$ ), ou à des projets tels Biblissima $(<$ http://www.biblissimacondorcet.fr/ $>$ ). L'interopérabilité de ces bases constitue un atout majeur pour la recherche, permettant un passage rapide entre des contenus complémentaires. 


\section{SIGILLA : base numérique des sceaux conservés en France}

Initié en 2012 dans le cadre d'un partenariat scientifique entre les Archives départementales de la Vienne et le CESCM de Poitiers, le projet SIGILLA a pris effectivement vie l'année suivante, sous l'impulsion d'une équipe de chercheurs du « Groupement d'intérêt scientifique SOURCEM - Source de la culture européenne et méditerranéenne » visant l'élaboration d'une base de données nationale recensant les sceaux (empreintes et matrices) conservés ou documentés dans les collections françaises, publiques (archives nationales et départementales, bibliothèques, musées) comme privées (particuliers, sociétés savantes) ${ }^{12}$.

Maintenant porté par l'École Pratique des Hautes Études (Paris, EPHE-PSL) et piloté par un consortium réunissant des institutions de recherche et de conservation (CESCM-Université de Poitiers/CNRS, CNRS-IRHT, Centre Jean Mabillon-École Nationale des Chartes, CRAHAM-Université de Caen-Normandie, CRULHUniversité de Lorraine, Service interministériel des Archives de France, Archives Nationales), SIGILLA se propose de fédérer les initiatives de diffusion numérique des données sigillographiques, de promouvoir les recherches dans le domaine de la sigillographie, de favoriser la conservation de cette source, aujourd'hui menacée mais pourtant universelle et fondamentale de la culture européenne. Ne s'adressant qu'aux spécialistes, ce programme nourrit l'ambition de fournir aux chercheurs de tout domaine un outil performant leur donnant accès à une documentation vaste - les sceaux forment un des plus abondants corpus d'objets médiévaux - et capable d'éclairer des pans entiers de la culture et de la société de la fin du Moyen Âge et de la première époque moderne : ses modes de pensée, ses codes, ses mœurs, ses goûts artistiques, ses rapports sociaux et institutionnels.

Pour atteindre son but, SIGILLA vise, avant tout, à récolter et rendre disponibles les informations relatives à ces documents dispersés et seulement en partie accessibles à la consultation. Souvent les conditions de conservations des empreintes - qui sont des objets très fragiles - sont en effet un obstacle à l'examen direct des pièces et à leur déchiffrement. Jusqu'à des temps récents, les chercheurs intéressés à ce type de documents ont donc dû faire essentiellement appel à quelques inventaires papier, pour la plupart édités entre le $\mathrm{XIX}^{\mathrm{e}}$ et la première moitié du $\mathrm{XX}^{\mathrm{e}}$ siècle $^{13}$ : parfois incomplets et pas exempts d'erreurs, ils portent rarement - détail non négligeable - des illustrations graphiques ou photographiques des exemplaires analysés. Signe des temps qui changent, dans les dernières années ces outils de travails « traditionnels » sont accompagnés par de nombreuses mises en ligne de sceaux de la part des centres d'archives. Relevant d'une institution donnée, ces initiatives, bien que méritoires même sur le plan scientifique, ont toutefois la limite d'être consacrées à des collections restreintes, alors que leur caractère autonome entraîne une certaine dispersion de l'information, une différence de traitement des données et l'incompatibilité entre les bases réalisées ${ }^{14}$.

De ce constat est née l'idée d'une base de données nationale capable en même temps de moissonner et normaliser les données numé- 
riques existantes - sans dévaloriser pourtant le travail déjà accompli - et d'élargir le catalogage à d'autres collections. La mise au point d'un outil informatique capable de remplir cette double tâche a par conséquent constitué la première étape du projet, pour laquelle une longue phase de réflexion et d'échange entre les porteurs du projet a été nécessaire.

La création d'une plateforme numérique performante a en effet constitué le préalable pour la réussite d'un tel projet. D'abord, la quantité impressionnante de sceaux conservés ou connus par des sources indirectes (on parle de plusieurs centaines de milliers d'exemplaires pour la France et de plusieurs millions pour l'Europe) a obligé à prédisposer un travail d'inventaire et d'analyse se déroulant par étapes, de manière collaborative et s'étalant sur plusieurs années : seulement l'emploi d'un outil informatique aurait donc permis de rendre accessible à tous, à mesure de leur collecte, l'ensemble des données sigillographiques conservées (matrices, empreintes, moulages, dessins et photographies) et d'élargir progressivement le terrain d'action du programme avec l'introduction de nouveaux partenaires. Par-delà les avantages qu'une base de données relationnelle offre en termes de respect d'un protocole d'analyse établi, de mise à jour et de repérage des informations, de réalisation de recherches croisées et d'interopérabilité des données, elle permet également de coupler la description des sceaux à des images normalisées et en haute définition, offrant ainsi aux utilisateurs l'opportunité d'examiner dans le détail les exemplaires recensés. Enfin, l'outil numérique permet de marquer les distances par rapport aux catalogues traditionnels de sceaux par le biais de nouvelles applications qui ouvrent l'objet sceaux à de nouvelles perspectives de recherche : un «module héraldique » particulièrement innovant qui permet de renseigner et de rechercher via les composantes de l'image (couleurs, partitions, meubles) les informations relatives aux armoiries présentes sur le sceau, source essentielle de l'héraldique ; la numérisation 3D des sceaux, par photogrammétrie ou par tomographie, qui restitue la matérialité d'un objet en volume trop souvent considéré en deux dimensions ; la modélisation d'une matrice virtuelle à partir des empreintes conservées ; la création d'un module SIGISCRIPT consacré au catalogage semi-automatisé de l'épigraphie du sceau et à la recherche numérique sur cette source encore largement sous-exploitée.

La phase préliminaire de concertation a été également consacrée à l'élaboration d'une fiche type de description qui, tout en assurant l'ergonomie de la base, permet de classer les nombreuses informations qu'un sceau peut transmettre (et que 1'utilisateur de la base peut espérer trouver) ${ }^{15}$ : sigillographiques, héraldiques, diplomatiques, prosopographiques, artistiques. La base propose d'ailleurs un mode d'approche inédit à cette source portant la description non plus sur l'empreinte, mais sur la matrice originelle ou, à défaut, sur le prototype sigillaire à l'origine des empreintes multiples. La base énumère donc, pour chaque sigillant, l'ensemble des sceaux-type dont il a fait usage, tout en fournissant un catalogue aussi exhaustif que possible des empreintes tirées de chaque exemplaire, quelqu'en soit la forme (empreinte de cire, bulle, sceau sous papier, cachet ou timbre à sec), le média de transmission (empreinte originale, moulage, dessin, cliché 
photographique), l'état et le lieu de conservation. Même si la priorité est donnée aux sceaux du Moyen Âge, aucune limite chronologique n'a été imposée à la collecte, pour que les partenaires du projet puissent verser les données en fonction de la nature de leurs fonds.

Librement consultable en ligne depuis mars 2016 et hébergée par la TGIR HUMA-Num, SIGILLA compte à ce jour - mais les chiffres sont en augmentation constante - près de 5000 sceaux de référence (sceaux-types) illustrés par leurs multiples empreintes connues (plus de 6988) et les actes auxquels elles sont appendues (environ $6345)^{16}$. Le nombre d'items recensés est toutefois en évolution rapide et constante grâce à une alimentation régulière de la base par plusieurs acteurs, opérant à la fois sur le moissonnage des données déjà en ligne, par la rétro-conversion des catalogues papiers et par la saisie de sceaux inédits. Pour permettre l'avancement rapide de ce travail et assurer l'uniformité des données versées dans la base, une interface de saisie a été mise au point. Conçue pour être le plus intuitive possible, elle constitue un compromis entre la qualité scientifique de l'information collectée, les attentes des utilisateurs et les capacités réelles des agents qui sont (et seront) appelés à alimenter la base. Il faut en effet tenir compte que ces derniers ne seront pas nécessairement des spécialistes en sigillographie (relativement rares), mais des personnes (étudiants universitaires, doctorants, conservateurs, chercheurs) formées au fur et à mesure de l'avancement du projet par des stages tenus par les membres du comité de pilotage. Le formulaire de saisie est formé par des champs, en partie complétés à partir de listes fermées accessibles pas des menus déroulants, consacrés aux informations diplomatiques et archivistiques sur le document, à celles sur le sigillant, à la description du sceau, à la bibliographie, à l'iconographie (chaque entrée est illustrée par des photographies du sceau et de l'acte auquel il est attaché) et à l'héraldique. Les données ainsi collectées sont validées par les webmasters de la base avant d'être mises en ligne.

La base des sceaux est consultable via le site internet SIGILLA, à accès libre et gratuit $(<$ www.sigilla.org $>)$. Réalisé en tenant un juste milieu entre le confort d'utilisation, l'esthétique et la teneur scientifique des contenus, le site donne accès aux données collectées par le biais d'un moteur de recherche (" rechercher un sceau »). Les résultats obtenus renvoient à deux types de fiches : les « fiches sigillant», qui réunissent tous les types de sceaux utilisés par une personne physique ou morale donnée (on y trouve également les informations biographiques, généalogiques et institutionnelles, comme la liste des sceaux-type connus) ; les fiches « sceautype », réunissant les informations relatives à la matrice originale ou, quand celle-ci est perdue, celles livrées par les empreintes connues tirées d'une même matrice. Ces dernières portent les informations sigillographiques sur l'exemplaire concerné (date d'utilisation, forme, dimension, description, contenu et langue de l'inscription), suivies par la liste exhaustive des occurrences connues. Ordonnés hiérarchiquement en vertu de leur degré de proximité à la matrice (empreinte, sceau sur papier, tirage dans le bronze ou surmoulage, dessin d'érudits, moulage, cliché photographique), ces documents sont analysés dans des notices détaillées recensant l'ensemble des informations recueillies lors de la saisie : 
cote de l'acte, matière, couleur, état matériel, type d'attache. Chaque notice est complétée par un cliché du sceau et, le cas échéant, par une couverture photographique de l'exemplaire en trois dimensions.

Revenant à la page d'accueil, l'utilisateur de la base peut également trouver des liens vers un espace pédagogique présentant l'étude des sceaux et les outils de base ; une présentation des auteurs de la base ; une rubrique consacrée à la description (une fois par mois) d'un sceau inédit ou remarquable par son histoire, sa composition ou son iconographie (rubrique Le sceau $d u$ mois). Une section « actualités » informe les internautes des nouveautés concernant l'avancement du projet, les activités de ses partenaires, l'organisation de colloques et journées d'étude, le développement de programmes de recherche complémentaires par d'autres institutions.

Financé par de divers institutions et programmes (SIAF, consortium COSME, fondations privées et mécénat), SIGILLA vise maintenant, dans le cadre du DIM, à concentrer son action sur les collections régionales de l'Île-de-France et notamment sur les 60000 moulages de sceaux du Service des sceaux des Archives Nationales et sur les dizaines de milliers de sceaux des Pièces originales de la Bibliothèque nationale de France. La mise en ligne de ce corpus imposant révolutionnera sans doute les recherches sigillographiques, mettant à disposition des chercheurs une masse documentaire inégalée par sa consistance et variété. Cette mission sera accompagnée par une extension de la collecte à l'échelle nationale et par le renforcement du rayonnement du programme à l'international.
D'un côté, une vingtaine de conventions ont été déjà signées ou sont en cours de signature avec des Archives départementales ou municipales (Aube, Côte-d'Or, Lozère, Haute-Savoie, Vienne, Joigny, Briançon, Dijon, Remiremont, Semur-enAuxois, Provins) ; dans ce cadre, les séances de formation proposées aux partenaires du projet et aux étudiants universitaires collaborateurs assureront l'avancement rapide de l'enrichissement de la base. De l'autre, un ambitieux projet transfrontalier de collecte des sceaux de la Savoie historique (INTERREG SAVOIE) est à l'étude avec les conservateurs français (Archives départementales de la Haute-Savoie, de Savoie et de la Côte d'or), suisses (Archives cantonales de Genève et de Lausanne, archives de l'abbaye de Saint-Maurice d'Agaune) et italiens (Archivio di Stato de Turin et Musées de Turin) : son actuation est liée au succès de la demande de financement qui a été déposée dans le cadre des programmes au soutien des actions de coopération européenne à l'échelle du massif alpin.

La réalisation de ce projet permettrait d'affirmer le protocole d'analyse du sceau élaboré pour SIGILLA comme une référence à un niveau international et de faire évoluer, sur le moyen et le long terme, la base comme une véritable plateforme commune pour la numérisation des collections de sceaux du continent (à ce jour la base Sigilla offre déjà la plus grande collection européenne de sceaux librement accessible en ligne ${ }^{17}$ ).

\section{ArmmA : l'Armorial Monumental du Moyen Âge}

Dernier né parmi les projets faisant maintenant partie de la famille E-SIGNA, le programme 
ArmmA, Armorial Monumental du Moyen Âge, propose un inventaire systématique et une étude analytique des décors héraldiques monumentaux produits en France entre le milieu du XII et le milieu du XVI ${ }^{e}$ siècle. Sans appliquer aucune distinction d'ordre qualitatif, matériel ou lié à l'état de conservation des œuvres répertoriées, l'étude s'attache à la fois aux ensembles héraldiques conservés in situ, à ceux disparus mais documentés par les sources indirectes et aux pièces erratiques, à savoir celles sorties de leur contexte d'origine et conservées, pour la plupart, dans les collections muséales. ArmmA a été d'ailleurs conçu dès l'origine avec un double objectif : scientifique et patrimonial. D'un côté, il s'agit en effet de contribuer à la connaissance des fonctions de l'image héraldique dans l'espace monumental par le biais d'un travail d'analyse conduit sur un corpus large et très varié, palliant ainsi une vraie lacune dans $l^{\prime}$ historiographie ${ }^{18}$. De l'autre, on souhaite contribuer à la valorisation d'un ensemble d'images, souvent méconnu et plus facilement exposé au danger de l'abandon et des restaurations « abusives », à travers l'attribution et la datation des attestations héraldiques inventoriées et la communication des résultats de la recherche auprès aussi des acteurs de la conservation et du large public.

Issu d'un travail exploratoire mené en 2013 par Laurent Hablot sur le patrimoine héraldique de la région Poitou-Charentes, le programme ArmmA a été par la suite développé au CESCM de Poitiers, à partir de janvier 2014, par Matteo Ferrari dans le cadre d'abord d'une bourse postdoctorale de l'Université de Poitiers, puis de contrats de recherche financés par la DRAC Poitou-Charentes (maintenant Nouvelle-Aquitaine). Dans le cadre de cette mission, étalée sur 26 mois non continus, il a été dressé un inventaire exhaustif des documents héraldiques monumentaux conservés ou documentés dans une aire géographique qui correspond au Poitou historique. Alors que seulement une cinquantaine de décors héraldiques était connue d'après la bibliographie consacrée au patrimoine artistique régional, 420 " unités monuments 》 - à savoir des édifices caractérisés par la présence d'au moins un élément héraldique d'époque médiévale - ont été recensées, dont 300 uniquement dans le département de la Vienne (61 dans le seul Poitiers !). Cette somme déjà considérable ne reflète toutefois que partiellement le nombre de décors héraldiques réellement recensés. Un même édifice peut en effet contenir plusieurs " unités héraldiques », c'est-à-dire plusieurs ensembles d'armoiries cohérents et autonomes du point de vue de la réalisation et de la fonction. Au terme de la première mission de recherche (avril 2017), cet ensemble s'élevait déjà à presque 800 occurrences, pour un total estimé d'environs 2000 armoiries.

Par-delà l'aspect quantitatif, ce premier volet du programme a fourni surtout le terreau propice pour définir une méthodologie de travail, couplant l'action d'inventaire à l'étude analytique des occurrences répertoriées, examinées en relation à leur contexte de production et d'exposition. Le travail de documentation sur le terrain a été ainsi un préalable à l'étude historique, permettant non seulement de documenter les décors connus et d'en découvrir souvent d'inédits, mais aussi d'examiner quelle relation, physique et fonctionnelle, entretenait l'image héraldique avec son contexte architectural d'exposition ; d'évaluer son rapport éventuel avec d'autres types de décor 
(durables ou éphémères) ; d'estimer son impact visuel ; de reconnaître la présence de rapports hiérarchiques entre les armoiries représentées et d'en comprendre la signification. Il a été ainsi souvent possible d'extraire des informations inédites sur les espaces et leurs fonctions, ainsi que sur les intentions du commanditaire et sur l'histoire patrimoniale de l'édifice.

Les résultats issus de ce travail de documentation et d'analyse ont été réunis dans des notices monographiques, consacrées à chaque monument examiné ou, dans les cas les plus complexes, à chaque « unité héraldique » répertoriée ${ }^{19}$. Au fur et à mesure de l'avancement du projet, les notices ont été organisées et rendues disponibles dans une base de connaissances intégrée dans un site internet à accès libre et gratuit. Élaboré au sein d'une convention spécifique signée en 2014 entre le CESCM et le service informatique I-Médias de l'Université de Poitiers, le site ArmmA a été mis en ligne en décembre 2016 après une phase de test d'environ un $\mathrm{an}^{20}$. Réalisé avec Wordpress, il se caractérise à la fois par la facilité d'usage pour les auteurs - la saisie des informations est réalisée par une interface composée par des champs ouverts ${ }^{21}$ et des champs fermés constitués par des listes préétablies ${ }^{22}$ - et pour la simplicité d'accès aux informations pour les usagers. Dans le but de créer un outil capable de mieux capter l'attention des internautes, l'aspect visuel du site a été particulièrement soigné.

L'accès aux études monographiques (« notices monument ») s'opère soit par le biais de la carte interactive affichée sur la page d'accueil du site (et par le moteur de recherche placé juste en dessous), soit par le biais des outils de recherche placés sur la gauche de l'écran. Dans ce dernier cas, la recherche s'effectue à la fois par index, sur la base des listes générées par les données saisies (il est donc possible d'interroger la base en fonction de la période de réalisation du décor, du support armorié, de la technique d'exécution, du personnage ou de la famille représentés), ou par des recherches en plein texte, soit dans l'intégralité des contenus du site, soit par termes du blason (le langage utilisé pour décrire les armoiries) parmi les occurrences héraldiques répertoriées.

Conçues comme des notices d'un catalogue, accompagnées par un riche répertoire d'images, les notices fournissent une description analytique et une interprétation de chacune des " unités » héraldiques répertoriées ${ }^{23}$. Se conformant à une structure homogène, les notices fournissent tout d'abord des informations générales sur l'histoire monumentale et patrimoniale du bâtiment, avant de passer à l'examen et à l'interprétation des éléments héraldiques qui le caractérisent (ou qui le caractérisaient). L'étude est complétée par un plan de l'édifice, permettant de repérer les armoiries répertoriées dans l'espace ; par une bibliographie ciblée, formée par les références utilisées dans l'analyse du décor en question; par une documentation photographique exhaustive, composée par une sélection de clichés pris au cours des missions de terrain ainsi que par des clichés historiques ou des images de croquis (essentiels pour documenter l'aspect originel des pièces perdues, abîmées, déplacées). Les articles monographiques sont associés à des fiches synthétiques qui proposent l'« édition » de chaque élément héraldique inventorié à son intérieur : sa description dans la langue héraldique (blasonnement); la mention d'éventuels ornements extérieurs de 
l'écu (cimiers, lambrequins, tenants, enseignes de fonction) ; l'identification de l'armoirie ; sa datation établie par des tranches d'un quart de siècle ; la description de son emplacement ; l'indication de la technique d'exécution ${ }^{24}$. Les fiches héraldiques sont complétées par l'image de l'armoirie dans son état actuel - ou documenté, quand elle est perdue - et par sa reconstruction graphique avec la réintégration éventuelle des meubles et des couleurs ${ }^{25}$.

Via la page d'accueil, l'utilisateur du site peut également avoir accès à d'autres contenus. Il trouve notamment un descriptif du projet et de ses missions ; une présentation des auteurs et des collaborateurs du programme; une bibliographie sélective sur l'héraldique et sur l'héraldique monumentale, conçue notamment pour orienter les néophytes à l'intérieur d'une production écrite de plus en plus riche mais de qualité très irrégulière. Dans cette section de la page d'accueil, un formulaire de contact est également disponible pour soumettre des questions aux responsables du projet, leur signaler des erreurs, des omissions ou des décors qui n'ont pas encore été répertoriés.

L'expérience acquise et les outils mis en œuvre dans la phase poitevine du projet constituent un atout majeur pour son extension à plus grande échelle. Cet élargissement de l'aire d'action d'ArmmA passe avant tout par la pérennisation du projet et par une évolution de la base déjà créée afin de mieux répondre aux exigences du public très varié concerné par ces données. L'intégration d'ArmmA dans le programme E-SIGNA et l'affiliation de ce dernier au DIM « Sciences du texte et connaissances nouvelles » viennent d'en relancer l'action, à travers l'ouverture d'un volet consacré à l'inventaire et à l'étude du patrimoine héraldique monumental de l'île-de-France. Si dans cette région les attestations héraldiques ont sûrement souffert, plus qu'ailleurs, des destructions révolutionnaires et des actions de renouvellement urbain opérées aux $\mathrm{XIX}^{\mathrm{e}}-\mathrm{XX}^{\mathrm{e}}$ siècles, les décors armoriés médiévaux demeurent nombreux. En outre, des fragments héraldiques provenant de monuments détruits - surtout des sculptures en pierre, mais aussi des fragments de vitraux ou des carreaux de pavement - ont été mis à l'abri dans les collections muséales (musée de Cluny, musée Carnavalet, musée d'art et d'histoire de Melun...), alors que les sources indirectes, textuelles et visuelles, documentent les innombrables décors perdus (c'est les cas des relevés de Gaignères qui offrent, par exemple, un aperçu des monuments funéraires armoriés, comme c'est le cas pour ceux de l'église parisienne de Saint-Martin-des-Champs ${ }^{26}$ ). Dans le cadre de la mission actuellement en cours 197 monuments héraldisés ont été déjà recensés, dont 49 dans le seul Paris (une vingtaine de notices est déjà disponible sur le site ArmmA).

La création de partenariats est également à l'étude pour élargir la couverture de la base à d'autres régions dont le « potentiel héraldique »a été déjà sondé, telles l'Auvergne et la Bretagne. Les DRAC régionales sont des interlocuteurs privilégiés et leur association au programme, en termes d'accès à la documentation et de financement de vacations. Leur collaboration constitue un préalable pour un développement du projet à l'échelle nationale. Sur le moyen terme, la base ArmmA se propose donc d'évoluer vers la forme d'une plateforme alimentée de manière participative, tout en restant soumise au contrôle d'une direc- 
tion stricte pour garantir la valeur scientifique des contenus et l'homogénéité des informations saisies. De cette manière sera possible de fédérer enfin la recherche sur l'héraldique monumentale médiévale et de pallier ainsi la fragmentation et la dispersion extrême des études dans ce domaine.

\section{Conclusion}

Associant les méthodes et la rigueur des sciences de l'érudition aux innovations apportées par les nouvelles technologies, le projet E-SIGNA vise donc à renouveler les études sur l'emblématique médiévale s'ouvrant à des corpus d'œuvres très larges, aujourd'hui finalement exploitables grâce aux humanités numériques. Fort d'une méthodologie de travail forgée au cours de ces dernières années et d'outils informatiques déjà performants, qui ont d'ailleurs rencontré l'appréciation de la communauté scientifique, E-SIGNA souhaite fournir un apport novateur à la réflexion sur les formes et les fonctions de l'emblématique médiévale s'appuyant également sur un réseau de partenaires et de collaborateurs ample et éprouvé.

Si les bases DEVISE, SIGILLA et ArmmA ont été d'abord conçues comme des outils de connaissance et de recherche historique, s'adressant à un public très large et varié, elles ont également l'ambition de contribuer à la conservation et à la mise en valeur d'un patrimoine souvent méconnu, fragile, se trouvant dans des conditions de conservations pas toujours optimales. Visant une couverture de l'emblématique médiévale à l'échelle nationale et sur ses différents supports, E-SIGNA se donne d'ailleurs pour objectif de coordonner les recherches dans ce domaine en proposant par le biais de ses bases - les premières de ce type à avoir été jusqu'à présent réalisées des plateformes informatiques à alimenter de manière participative, mais toujours sous un contrôle scientifique stricte.

Pour profiter pleinement du potentiel heuristique offert par l'informatique, le projet E-SIGNA souhaite renforcer l'interopérabilité entre ces bases et le connecter plus facilement à d'autres bases de connaissances développées au sein de programmes de recherches concernés par ces données, tels que les susmentionnées Bibale et Collecta. Réunis sous un même projet et complétés par les données issues d'autres expériences en cours en humanités numériques, les programmes DEVISE, SIGILLA et ArmmA- développées de façon inégale dans le temps et selon les moyens respectifs jusqu'à présent disponibles, mais strictement complémentaires - cumuleront leurs atouts et leurs outils (reconnaissance et catalogage automatique des données héraldiques, reconnaissance automatique d'images) et les perspectives de leurs données croisées, en termes de valorisation du patrimoine (identification, datation, lecture historique), d'études prosopographiques et généalogies, d'analyses formelles et stylistiques. 


\section{$R \cdot \dot{E} \cdot F \cdot E ́ R \cdot E \cdot N \cdot C \cdot E \cdot S$}

BAUDOT, Marcel « Une classification informatique des armoiries ", La Gazette des archives, 1, 105, 1979, p. 122 (http://www.persee.fr/doc/ gazar_0016-5522_1979_num_105_1_2677).

DUBUC, René, Essai de classification systématique des armoiries européennes, t. 1-3, Rochefort, 1977-1978 (La documentation cistercienne, 21).

HABLOT, Laurent, La devise, mise en signe $d u$ prince, mise en scène du pouvoir. Les devises et l'emblématique des princes en Europe à la fin du Moyen Age, thèse de doctorat, Université de Poitiers, 2001, dir. M. Pastoureau, M. Aurell.

HABLOT, Laurent, " Le programme 'Sigilla', base de données nationale des sceaux des archives françaises », dans M. Libert, J.-F. Nieus (dir.), Le sceau dans les Pays-Bas méridionaux, $X^{e}$-XVI siècles. Entre contrainte sociale et affirmation de soi, actes du colloque (Bruxelles-Namur, 27-28 novembre 2014), Bruxelles, 2017, p. 111-124 (Archives et bibliothèques de Belgique, numéro spécial, 103).

HABLOT, Laurent, Manuel d'héraldique et d'emblématique médiévale. (XII ${ }^{e}-X V I^{e}$ siècles), Tours, PUFR, 2019.

HABLOT, Laurent; HILTMANN, Torsten (dir.), Heraldry in the Medieval City : the case of Italy in the European context, actes du colloque (Rome, 5-7 mai 2015), sous presse.

HILTMANN, Torsten ; METELO DE SEIXAS, Miguel (dir.), Heraldry in Medieval and Early Modern State-Rooms, actes du colloque (Münster, 16-18 mars 2016), sous presse.

MÉRINDOL, Christian de, La maison des chevaliers de Pont-Saint-Esprit, t. II. Les décors peints : corpus des décors monumentaux peints et armoriés du Moyen Âge en France, Pont-SaintEsprit, Musée d'Art Sacré du Gard, 2001. MERCIER, Alain, La deuxième fille de Cluny. Grandeurs et misères de Saint-Martin-desChamps, Grenoble, Glénat/Le Cnam, 2012. MÉRINDOL, Christian de, Images du royaume de France au Moyen Âge. Décors monumentaux peints et armoriés. Art et histoire, Pont-SaintEsprit, éd. Gérard Louis, 2013.

PASTOUREAU, Michel, Traité d'héraldique, Paris, Picard, 1993 (éd. or. 1979). 
$\mathrm{N} \cdot \mathrm{O} \cdot \mathrm{T} \cdot \mathrm{E} \cdot \mathrm{S}$

1. Si cet article est le fruit d'un travail de collaboration entre les deux auteurs, l'introduction et le paragraphe consacré au projet ArmmA seront à attribuer à Matteo Ferrari, alors que les paragraphes sur les projets Sigilla et Devises et les conclusions seront à attribuer à Laurent Hablot.

2. Voir, par exemple, Dubuc 1977-1978, avec les notes de Baudot 1979.

3. Pastoureau 1993, p. 243, 310.

4. Le projet développé à Münster par le groupe coordonné par Torsten Hiltmann, par exemple, vise la construction d'un outil basé sur la reconnaissance automatisée des images. Dans ce même cadre, l'outil de recherche héraldique élaboré au sein du projet Sigilla (sur lequel voir infra) se fonde en revanche sur un système de dessin automatisé de l'armoirie. Il permet ainsi de s'affranchir de la recherche habituelle par blasonnement qui, demandant une certaine compétence lexicale, s'avère souvent difficile à maîtriser et inefficace à répondre aux nécessités des auteurs et des utilisateurs des bases héraldiques.

5. Voir, à titre d'exemple, la base des sceaux des Archives d'État de Turin, en Italie ( $<$ http://archiviodistatotorino.beniculturali.it/work/sig prg.php? $>$ ), l'inventaire des moulages des sceaux des Archives de l'État en Belgique, Bruxelles $(<$ https://search.arch. be/fr/rechercher-des-archives/resultats/ead/index/ eadid/BE-A0510 005990005868 FRE/open/c:2. >), l'inventaire et étude réalisés par Laura Cirri sur les armoiries des chevaliers de l'Ordre de Santo Stefano à Pise (< http://velasquez.sns.it/stemmi/index.php $>$ ) ou, encore, le catalogue héraldique Cermelli Papiani des Archives d'État de Florence ( $<$ http://www.archiviodistato.firenze.it/ceramellipapiani/ $>$ ).

6. Cf. Digitised Armorials : < https://heraldica. hypotheses.org $1770>$.

7. Hablot 2001.

8. Sur le sujet voir Hablot 2019.

9. La migration de la base DEVISE vers l'EPHE sera effectuée dans les mois prochains.

10. Les notices ne sont pas anonymes, elles sont toujours signées par leurs auteurs.

11. Par exemple : Maison de Castille $>$ Isabelle de Castille/Isabelle la Catholique $>$ la grenade ; les lettres F Y ; l'aigle nimbé, les couleurs vert/bleu; le faisceau de flèches.

12. Ce groupe était formé par Jean-Christophe Blanchard (CRULH, Nancy), Laurent Hablot (CESCM, Poitiers), Michel Pastoureau (EPHE, Paris), Hanno Wisjman (IRHT, Paris) : cf. Hablot 2017.

13. L. Douet d'Arcq, Collection de sceaux des archives de l'Empire, Paris, 1867 ; A. Coulon, Inventaire de sceaux de la Bourgogne, Dijon 1912 ; F. Eygun, Sigillographie du Poitou jusqu'en 1515, Poitiers, 1938.

14. Parmi les archives qui ont mis en ligne leurs collections de sceaux on citera celles de l'Aube, de Loire-Atlantique, de la Creuse, de l'Yonne, de la Somme, de la Sarthe et du Puy-de-Dôme. Des projets de numérisations sont également conduits par le Cabinet des Médailles de la Bibliothèque nationale de France (projet Matrice et Chartes scellées) et par les Archives Nationales (sceaux plaqués du haut Moyen Âge). Des bases de données sigillographiques sont également développées à l'étranger (Allemagne, Portugal, Royaume-Uni).

15. Le formulaire de description proposé par SIGILLA tient compte de la note $\operatorname{ISAD}(\mathrm{G})$ établie per les Archives Nationales en 2005. Ne se contraignant pas strictement à ces consignes, il les adapte et les simplifie en fonction de la finalité de la base.

16. 4678 sigillants ont été recensés jusqu'à présent (données mises à jour le 10/12/2018).

17. Si la collection en ligne des moulages des sceaux 
des Archives d'État de Belgique présente presque 38000 unités, elle n'offre que l'identification de l'exemplaire, sa datation sur la base du document auquel il est attaché et une image de petite taille $:<\underline{\text { https: } / /}$ search.arch.be/fr/rechercher-des-archives/resultats/ ead/index/eadid/BE-A0510_005990_005868_FRE/ node/c:0.c:10.\#c:0.c:10 >. Quant à eux, les sites déjà opérationnels en France fournissent, en total, environ 10000 entrées.

18. Mises à part les études de Christian de Mérindol (voir surtout Mérindol 2001 et 2013) et d'autres rares chercheurs, les décors héraldiques monumentaux ont suscité moins d'attention de la part des spécialistes, tout étant souvent considérés comme de simples ornements par les historiens de l'art. Le nouvel intérêt suscité par ce type de documents est maintenant prouvé par les volumes collectifs Hablot, Hiltmann sous presse et Hiltmann, Seixas sous presse.

19. Dans une église, par exemple, chaque chapelle seigneuriale fait l'objet d'une notice autonome.

20. La migration de la base, actuellement stockée à l'Université de Poitiers, vers l'EPHE sera effectuée dans les mois prochains.

21. Il s'agit des champs consacrés à l'analyse du monument, au blasonnement de l'armoirie et à la bibliographie.

22. Concernant l'état de conservation de l'édifice, sa destination d'usage, l'emplacement de l'image héraldique, son support, la technique de réalisation, sa datation.

23. Cette section du site comptait 260 pages au $10 / 12 / 2018$.

24. La base Armma compte à ce jour (10/12/2018) 972 fiches armoiries.

25. À ce propos voir le travail d' « édition » réalisé pour les armoiries presque illisibles à l'œil nu repérées dans l'église de Châtillon-sur-Thouet ( $<\underline{\text { http:// }}$ base-armma.edel.univ-poitiers.fr/monument/eglise- de-la-madeleine-maison-dieu-chatillon-sur-thouet/ >, cons. 30/5/2018) ou dans l' « Hôtellerie » de l'abbaye de Nanteuil-en-Vallée ( $<$ http://base-armma.edel. univ-poitiers.fr/monument/abbaye-saint-benoitet-sainte-marie-hotellerie-nanteuil-en-vallee/ >, cons. 30/5/2018). La reconstruction graphique des armoiries a été pour le moment assurée par l'utilisation de logiciels de dessin héraldique en open-accès, tel Héraldique 8.0. Elle sera bientôt remplacée par le susmentionné outil de dessin et de recherche héraldique mis au point dans le cadre du projet Sigilla.

26. Sur lesquels voir Mercier 2012. Dans le cadre d'E-SIGNA un partenariat avec le projet Collecta. Archives numériques de la collection Gaignières $(<\underline{\text { www.collecta.fr }}>)$, dirigé par Anne Ritz-Guilbert, a été établi. 
$R \cdot E ́ \cdot S \cdot U \cdot M \cdot E ́$

Crée en 2018, E-SIGNA réunit trois projets concernant l'emblématique médiévale, développés de manière autonome mais complémentaire à partir de 2012 et associés à trois bases de connaissances, déjà accessibles en ligne : ArmmA est consacrée aux décors héraldiques monumentaux produits dans la France médiévale ; DEVISE est dédiée à l'emblématique européenne ; SIGILLA collecte les sceaux conservés en France. Associant la rigueur des sciences de l'érudition aux possibilités heuristiques et de communication offertes par les nouvelles technologies, E-SIGNA propose de revisiter avec méthode et perspective ces objets surreprésentés durant le Moyen Âge mais pourtant aujourd'hui encore très largement négligés : les armoiries, les devises, les sceaux.

\begin{abstract}
Created in 2018, E-SIGNA brings together three projects concerning the medieval emblematic, developed independently but complementary from 2012 and associated with three databases, already available online: ArmmA, dedicated to the monumental heraldry produced in medievalFrance; DEVISE, dedicated to the European badges and emblems; SIGILLA, collecting the seals preserved in France. Combining the rigor of the erudite knowledge with the heuristic and communication tools offered by the new technologies, E-SIGNA proposes to revisit, with method and perspective, those objectsoverrepresented during the European Middle Ages, but nevertheless largely neglected today: the coats of arms, the badges, the seals.
\end{abstract}

